\title{
Genome-Wide Mapping of Quantitative Trait Loci Conferring All-Stage and High-Temperature Adult-Plant Resistance to Stripe Rust in Spring Wheat Landrace PI 181410
}

\author{
Yan Liu ${ }^{1}$, Yanmin Qie ${ }^{1,2}$, Xing Li ${ }^{1,3}$, Meinan Wang ${ }^{1}$ and Xianming Chen ${ }^{1,4, *}$ \\ 1 Department of Plant Pathology, Washington State University, Pullman, WA 99164-6430, USA; \\ y.liu@wsu.edu (Y.L.); qieyanmin2014@163.com (Y.Q.); lixing@hebau.edu.cn (X.L.); \\ meinan_wang@wsu.edu (M.W.) \\ 2 Institute of Cereal and Oil Crops, Hebei Academy of Agricultural and Forestry Sciences, 162 Hengshan \\ Street, Gaoxin District, Shijiazhuang, Hebei 050035, China \\ 3 College of Plant Protection, Hebei Agricultural University, Baoding, Hebei 071001, China \\ 4 US Department of Agriculture, Agricultural Research Service, Wheat Health, Genetics, and Quality Research \\ Unit, Pullman, WA 99164-6430, USA \\ * Correspondence: xianming.chen@usda.gov; Tel.: +1-509-335-8086
}

Received: 20 December 2019; Accepted: 9 January 2020; Published: 12 January 2020

\begin{abstract}
Stripe rust, caused by Puccinia striiformis f. sp. tritici (Pst), is one of the most destructive diseases of wheat in the world. Genetic resistance is the best strategy for control of the disease. Spring wheat landrace PI 181410 has shown high level resistance to stripe rust. The present study characterized the landrace to have both race-specific all-stage resistance and nonrace-specific high-temperature adult-plant (HTAP) resistance. To map quantitative trait loci (QTL) for the resistance in PI 181410, it was crossed with Avocet S (AvS), from which a recombinant inbred line population was developed. The $\mathrm{F}_{5}-\mathrm{F}_{8}$ populations were consecutively phenotyped for stripe rust response in multiple field environments under natural Pst infection, and the $\mathrm{F}_{7}$ population was phenotyped in seedlings at low temperature and in adult-plant stage with selected Pst races in the greenhouse. The $\mathrm{F}_{7}$ population was genotyped using the $90 \mathrm{~K}$ wheat SNP chip. Three QTL, QYrPI181410.wgp-4AS, QYrPI181410.wgp-4BL, and QYrPI181410.wgp-5BL.1, from PI 181410 for all-stage resistance, were mapped on chromosome arms 4AS, 4BL, and 5BL, respectively. Four QTL, QYrPI181410.wgp-1BL, QYrPI181410.wgp-4BL, QYrPI181410.wgp-5AS, and QYrPI181410.wgp-5BL.2, were identified from PI 181410 for HTAP resistance and mapped to 1BL, 4BL, 5AS, and 5BL, respectively. Two QTL with minor effects on stripe rust response were identified from AvS and mapped to 2BS and 2BL. Four of the QTL from PI 181410 and one from AvS were potentially new. As the 4BL QTL was most effective and likely a new gene for stripe rust resistance, three kompetitive allele specific PCR (KASP) markers were developed for incorporating this gene into new wheat cultivars.
\end{abstract}

Keywords: disease resistance; Puccinia striiformis; QTL mapping; stripe rust; Triticum aestivum; wheat

\section{Introduction}

Wheat (Triticum aestivum L.) is one of the most important food crops worldwide, as it provides humans most of their daily calories and proteins. With the growing of the human population, wheat demand is expected to increase by $66 \%$ in 2040 [1]. However, wheat production has been continually threatened by abiotic and biotic stresses. Stripe rust (also known as yellow rust), caused by fungal pathogen Puccinia striiformis Westend. f. sp. tritici Erikss. (Pst), is especially a devastating disease 
in the world [2,3], and the fungus is ranked among the global burden pathogens and pests of major crops [4]. The disease can cause more than $90 \%$ yield losses in fields grown with susceptible cultivars if the weather conditions are extremely favorable to stripe rust development [5].

Growing resistant cultivars and application of fungicides are two major approaches for control of stripe rust. Use of fungicides adds significant cost to production; has potential to select fungicide-tolerant populations of the pathogen; is potentially harmful to humans, animals, and the environment; and therefore, is limited in organic farming and areas under protection for wildlife habitats. In contrast, growing resistant cultivars does not have these problems, but is easy to use, efficient, and more effective for control of stripe rust [6,7]. On the other hand, resistance may have some other problems. For example, resistant cultivars may become susceptible to new virulent races of the pathogen or the resistance level may not be adequate when the disease is severe. However, these problems can be solved by developing cultivars with durable, high-level resistance [5].

Resistance to stripe rust is generally described in two types: all-stage resistance and high-temperature adult-plant (HTAP) resistance [2,7]. All-stage resistance (ASR), also called seedling resistance, is normally controlled by a single gene that provides high-level resistance throughout the growth stages. However, ASR is usually race-specific, and cultivars with this type of resistance can be easily overcome by new virulent races of the pathogen. More than 300 Pst races have been identified from wheat stripe rust samples collected from the US and several countries, and the recent predominant races in the US are virulent on 16 of the $18 Y r$ single-gene lines in the Avocet background that are used to differentiate Pst races [8-10]. In contrast, HTAP resistance, which is expressed when the plant grows old and weather becomes warm, is nonrace-specific and durable $[7,11]$. The quantitative nature makes HTAP resistance breeding relatively difficult compared to ASR, and also often the partial resistance may not provide adequate control if the disease starts in the early growth stage and the weather conditions favor the disease but do not favor expression of HTAP resistance. Therefore, it is better to combine both types of resistance by pyramiding genes for effective ASR and HTAP resistance to utilize their advantages and overcome the disadvantages. This approach requires a large number of genes with user-friendly markers for marker-assisted selection (MAS). Thus, mapping new resistance genes is a continual task for developing wheat cultivars with durable, high-level resistance to stripe rust to achieve sustainable and more effective control.

Wheat landraces are valuable sources of resistance to stripe rust. PI 181410, a spring wheat landrace originally from Afghanistan (https://npgsweb.ars-grin.gov/gringlobal/accessiondetail.aspx? $\mathrm{id}=1157002)$, has shown excellent resistance to stripe rust [12]. However, the genes for resistance to stripe rust in PI 181410 were not known. Therefore, the objectives of the present study were to (1) characterize stripe rust resistance in PI 181410, (2) identify and map genes or quantitative trait loci (QTL) conferring resistance, and (3) develop molecular markers for marker-assisted selection.

\section{Results}

\subsection{Characterization of Stripe Rust Resistance in PI 181410}

In the field, PI 181410 has always shown resistance with infection type (IT) 1-2 and severity (SE) $5 \%-20 \%$ to stripe rust at adult-plant stage in both Mount Vernon and Pullman, Washington, USA for 13 years since 2006 (Figure S1). In contrast, the susceptible check, AvS, has been always highly susceptible (IT 8, SE $90 \%-100 \%$ ) in the same nurseries. In the greenhouse tests, the seedlings of PI 181410 were resistant (IT 3) to Pst race PSTv-4, moderately resistant (IT 5) to PSTv-14, and susceptible (IT 8) to races PSTv-37, PSTv-40, and PSTv-51 in the low-temperature (LT) $\left(4-20^{\circ} \mathrm{C}\right)$ tests (Table 1 ). In the high-temperature (HT) $\left(10-30^{\circ} \mathrm{C}\right)$ tests, PI 181410 seedlings were highly resistant (IT 2) to PSTv-4, PSTv-14, PSTv-40, and PSTv-51 but susceptible (IT 7) to PSTv-37. The adult-plants of PI 181410 showed high resistance (IT 2) to all the five tested races PSTv-4, PSTv-14, PSTv-37, PSTv-40, and PSTv-51 at both LT and HT. For comparison, the seedlings and adult plants of AvS were always highly susceptible 
(IT 8-9, SE 80\%-100\%) to all tested races at both LT and HT. The results indicated that PI 181410 has both race-specific ASR and nonrace-specific HTAP resistance.

Table 1. Stripe rust infection types of PI 181410 to five predominant or most virulent races of Puccinia striiformis f. sp. tritici in the United States tested in seedling and adult-plant stages at low $\left(4-20^{\circ} \mathrm{C}\right)$ and high $\left(10-30{ }^{\circ} \mathrm{C}\right)$ temperature profiles.

\begin{tabular}{ccccccc}
\hline \multirow{2}{*}{ Growth Stage } & \multirow{2}{*}{ Temperatur Profile } & \multicolumn{4}{c}{ Infection Type $^{\text {a }}$} \\
\cline { 3 - 7 } & & PSTv-4 & PSTv-14 & PSTv-37 & PSTv-40 & PSTv-51 \\
\hline \multirow{2}{*}{ Seedling } & Low & 3 & 5 & 8 & 8 & 8 \\
\multirow{3}{*}{ Adult-plant } & High & 2 & 2 & 7 & 2 & 2 \\
& Low & 2 & 2 & 2 & 2 & 2 \\
\hline
\end{tabular}

${ }^{\text {a }}$ For comparison, AvS had infection type 8 or 9 in all of the tests.

\subsection{Stripe Rust Phenotypes of the Recombinant Inbred Line (RIL) Population}

The distributions of seedling IT among the $170 \mathrm{~F}_{7}$ RILs tested with both PSTv-4 and PSTv-14 were continuous, and the patterns were similar (Figure 1, Table S1). The $\mathrm{F}_{5}, \mathrm{~F}_{6}$, and $\mathrm{F}_{7}$ RILs were tested under natural infections of Pst in the fields at Mount Vernon and Pullman in 2015, 2016, and 2017, respectively. The IT distributions of adult plants among the 170 RILs in field environments were also continuous but the patterns are different (Figure S2). Similarly, the relative area under the disease progress curve (rAUDPC) data also had continuous distributions with slightly different patterns (Figure 2). These results indicated that the stripe rust resistance in PI 181410 was quantitatively inherited. However, the distribution patterns were similar between the races tested in the greenhouse and were different among the year-location environments.

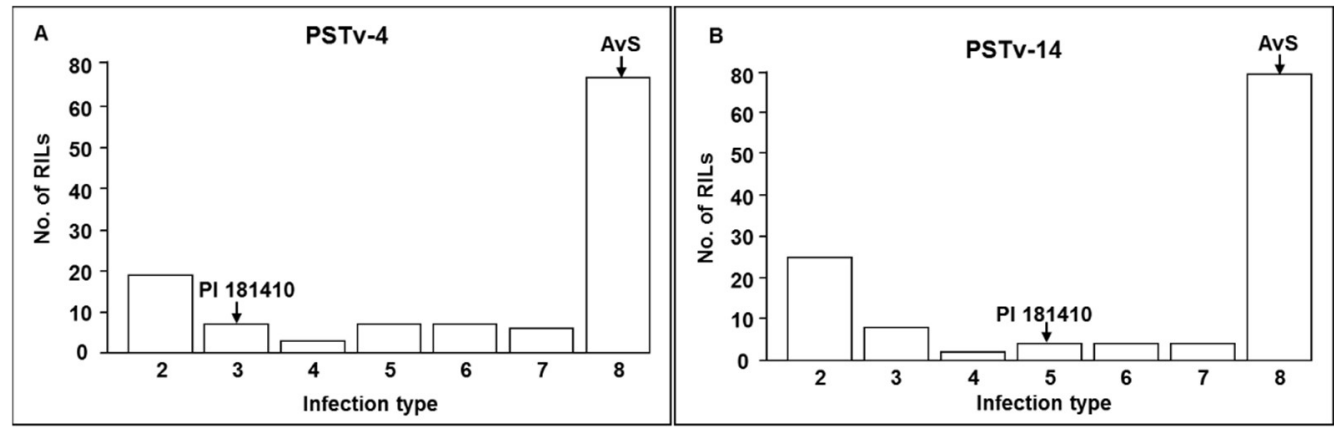

Figure 1. The distribution of infection types (ITs) among $\mathrm{F}_{7}$ recombinant inbred lines (RILs) derived from the cross AvS $\times$ PI 181410 tested in greenhouse using races (A) PSTv-4 and (B) PSTv-14 of Puccinia striiformis f. sp. tritici.

The analysis of variance (ANOVA) showed that the genetic and environmental effects and their interaction were all significant $(p<0.001)$ (Table 2$)$. Broad-sense heritability $\left(\mathrm{H}^{2}\right)$ was estimated as 0.68 for the rAUDPC data and 0.78 for IT data, indicating that the resistance in PI 181410 was highly heritable. The correlation coefficients of either IT or rAUDPC for all possible pairwise environment comparisons were highly significant $(p<0.001)$, but the values ranged from 0.29 to 0.79 (Table 3 ). Correlation coefficients were much higher between any two greenhouse tests or field tests than those between a field test and a greenhouse test. The phenotypical analyses indicated that different QTL controlled the resistances observed in different environments and suggested that QTL analysis should be used to map the genes controlling the resistance in the RIL population. 

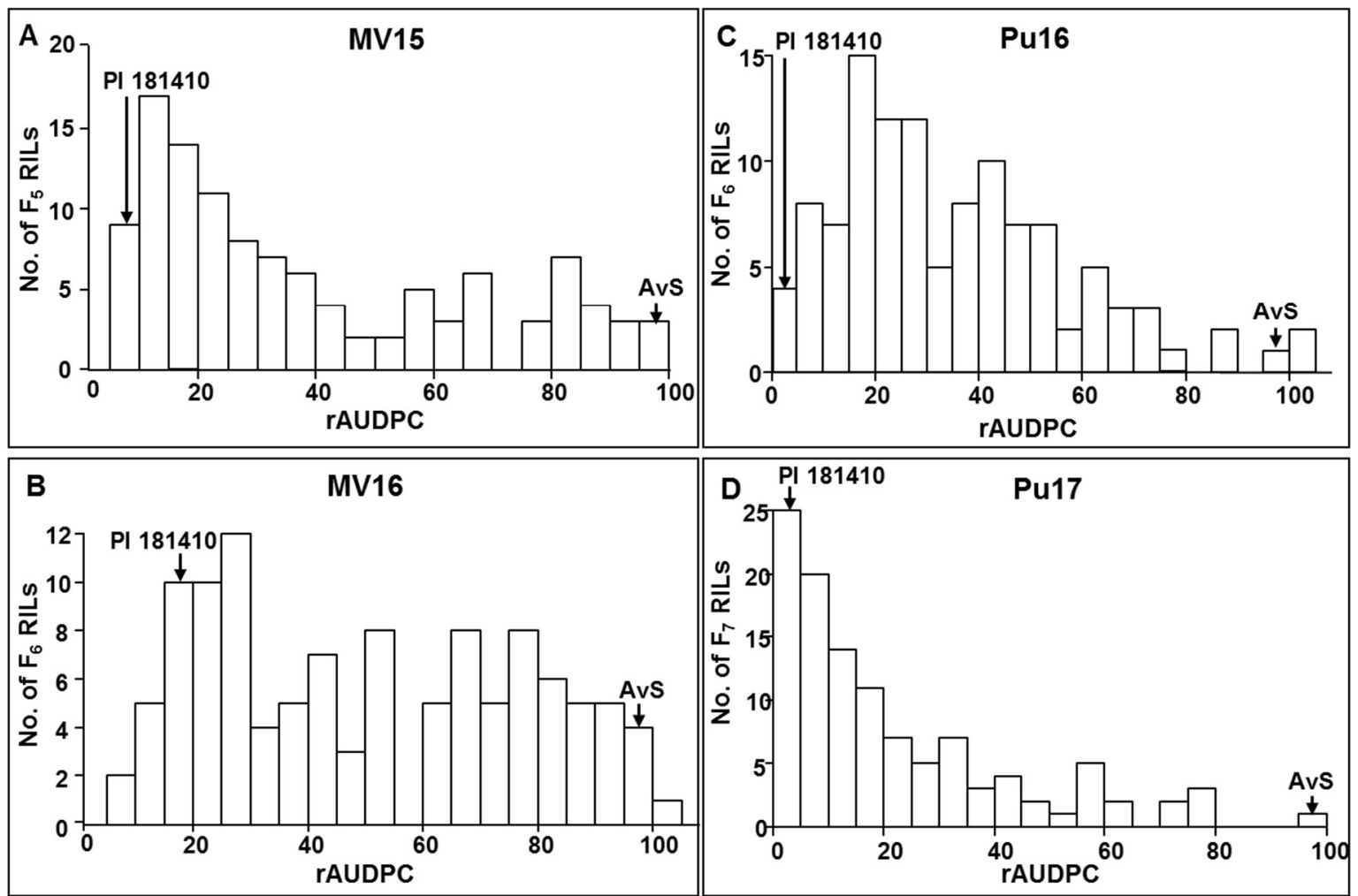

Figure 2. The distribution of relative area under the disease progress curve (rAUDPC) among recombinant inbred lines (RILs) derived from the cross AvS $\times$ PI 181410 in the field tests in Pullman and Mount Vernon, WA in 2015, 2016, and/or 2017.

Table 2. Analysis of variance (ANOVA) for relative area under the disease progress curve (rAUDPC) and infection type data for a recombinant inbred line (RIL) population derived from cross AvS $\times$ PI 181410 in different field environments of Pullman and Mount Vernon, WA in 2015, 2016, and/or 2017.

\begin{tabular}{ccccccccc}
\hline & \multicolumn{4}{c}{ rAUDPC } & \multicolumn{5}{c}{ Infection Type } \\
\hline Variance & $d f$ & MS & F & $p$ & $d f$ & MS & F & $p$ \\
\hline RIL & 162 & 4578 & 26.8 & $<0.0001$ & 162 & 48.7 & 43.6 & $<0.0001$ \\
Environment & 3 & 79480 & 465 & $<0.0001$ & 3 & 254 & 227.2 & $<0.0001$ \\
RIL $\times$ Environment & 485 & 671 & 3.93 & $<0.0001$ & 484 & 6.1 & 5.5 & $<0.0001$ \\
Error & 1268 & 171 & & & 1263 & 1.12 & & \\
\hline
\end{tabular}

Table 3. Correlation coefficients of infection type (IT) and relative area under the disease progress curve (rAUDPC) of the recombinant inbred lines of AvS $\times$ PI 181410 among the field tests in adult-plant stage in the fields under natural infections of Puccinia striiformis f. sp. tritici.

\begin{tabular}{ccccc}
\hline & \multicolumn{4}{c}{ Correlation Coefficient $^{\mathbf{b}}$} \\
\hline Test $^{\mathbf{a}}$ & MV15 & MV16 & Pu16 & Pu17 $^{* * *}$ \\
\hline MV15 & $0.80^{* * *}$ & $0.76^{* * *}$ & $0.69^{* * *}$ & $0.67^{* * *}$ \\
MV16 & $0.60^{* * *}$ & $0.86^{* * *}$ & $0.63^{* * *}$ & $0.57^{* * *}$ \\
Pu16 & $0.67^{* * *}$ & $0.69^{* * *}$ & $0.53^{* * *}$ & $0.61^{* * *}$ \\
\hline Pu17 & $0.67^{* * *}$ & $0.79^{* * *}$ & $0.68^{* * *}$ & $0.68^{* *}$ \\
\hline
\end{tabular}

${ }^{a} \mathrm{MV}=$ Mount Vernon and $\mathrm{Pu}=$ Pullman; and $15=2015,16=2016$, and $17=2017 .{ }^{\mathrm{b}}$ the left lower values are for comparing IT and the right upper values are for comparing rAUDPC values in different environments; and the diagonal four values are for comparing IT and rAUDPC in the same environments. ${ }^{\mathrm{C} * *}$ significant at $p<0.001$ and *** significant at $p<0.0001$. 


\subsection{Genetic Linkage Groups}

A total of 13,396 polymorphic single nucleotide polymorphism (SNP) markers were detected between parental lines AvS and PI 181410 using the 90K SNP chip. After removing SNPs that did not fit the 1:1 segregation ratio and duplicates of cosegregating SNPs, the final 3077 SNPs were used to construct linkage groups, and 20 linkage groups were obtained. The 20 linkage groups represent chromosomes 1A, 1B, 1D, 2A, 2B, 2D, 3A, 3B, 3D, 4A, 4B, 5A, 5B, 5D, 6A, 6B, 6D, 7A, 7B, and 7D (Table S2). The genetic map consisting of the 20 linkages is shown in Figure S3. The total genetic length was $2654 \mathrm{cM}$, and average genetic distance between two SNPs was $0.86 \mathrm{cM}$.

\subsection{QTL for ASR to Stripe Rust}

Using the two sets of IT data of $\mathrm{F}_{7}$ RILs at the seedling stage tested with races PSTv-4 and PSTv-14 in the greenhouse, we detected three QTL, QYrPI181410.wgp-4AS, QYrPI181410.wgp-4BL, and QYrPI181410.wgp-5BL.1, conferring resistance to both races (Table 4, Figure 3).

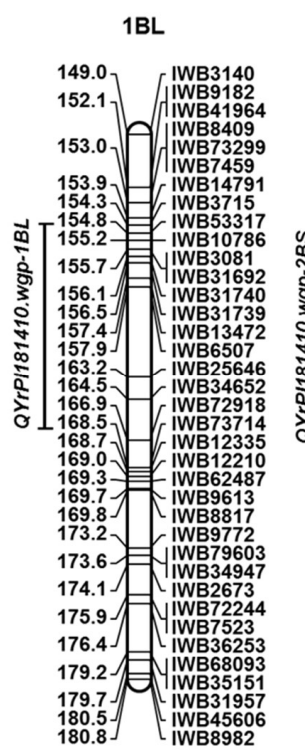

4BL

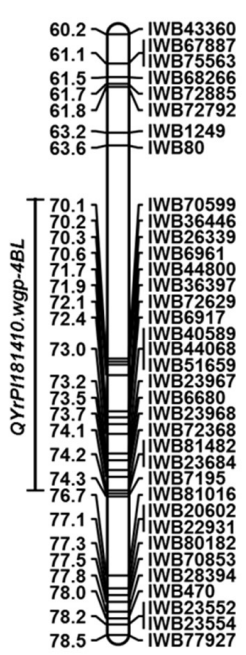

2BS

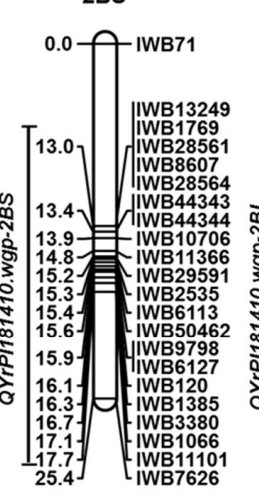

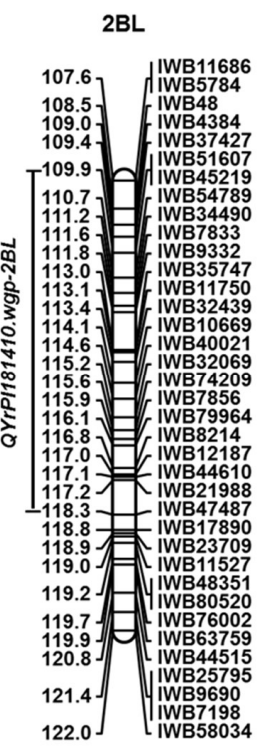

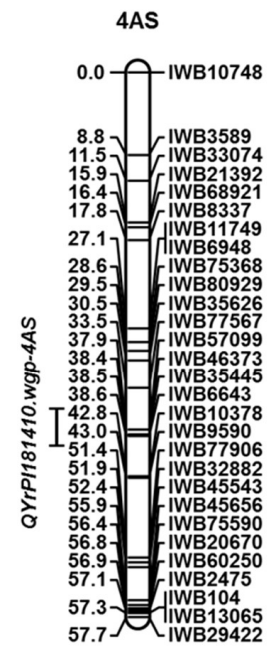

$5 B L$

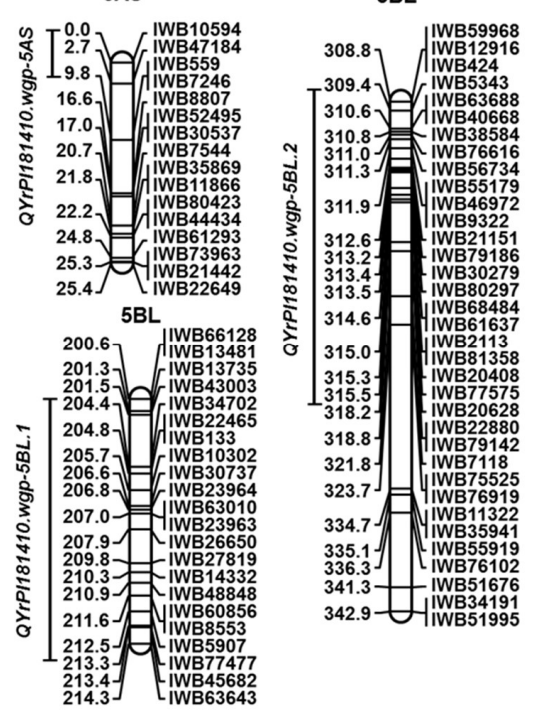

Figure 3. Genetic maps for the eight stripe rust resistance QTL identified in PI 181410 and AvS in this study. The solid bars on the left of each genetic map indicate the QTL position and interval. 
Table 4. Quantitative trait loci (QTL) associated with stripe rust all-stage resistance detected in wheat genotype PI 181410 using the recombinant inbred line population derived from AvS × PI 181410 .

\begin{tabular}{|c|c|c|c|c|c|c|c|}
\hline QTL & $\begin{array}{l}\text { Phenotypic } \\
\text { Data Set }^{\text {a }}\end{array}$ & $\begin{array}{l}\text { Closest } \\
\text { Marker }\end{array}$ & $\begin{array}{c}\text { Peak } \\
\text { Position }\end{array}$ & LOD $^{b}$ & $\begin{array}{l}\text { QTL Interval } \\
\text { (cM) }\end{array}$ & $\mathbf{R}^{2}(\%)^{\mathrm{c}}$ & $\begin{array}{c}\text { Additive } \\
\text { Effect }\end{array}$ \\
\hline \multirow[t]{2}{*}{ QYrPI181410.wgp-4AS } & PSTv-4-IT & IWB9590 & 43.0 & 3.3 & $41.0-45.0$ & $11.2^{* * *}$ & 0.5 \\
\hline & PSTv-14-IT & IWB9590 & 43.0 & 2.7 & $40.0-44.0$ & $5.4^{* *}$ & 0.4 \\
\hline \multirow{2}{*}{ QYrPI181410.wgp-4BL } & PSTv-4-IT & IWB6961 & 71.0 & 3.6 & $68.0 .0-88.0$ & $8.6^{* *}$ & 0.5 \\
\hline & PSTv-14-IT & IWB6961 & 71.0 & 2.5 & $70.0-90.0$ & $11.0^{* *}$ & 0.8 \\
\hline \multirow{2}{*}{ QYrPI181410.wgp-5BL.1 } & PSTv-4-IT & IWB73753 & 211.0 & 4.7 & $203.0-213.0$ & $15.1^{* * *}$ & 0.6 \\
\hline & PSTv-14-IT & IWB73753 & 211.0 & 4.5 & $203.0-213.0$ & $14.0^{* * *}$ & 0.7 \\
\hline
\end{tabular}

${ }^{a}$ In the phenotypic data sets, IT = infection type, the disease reactions of RILs were tested using two races, PSTv-4 and PSTv-14, in greenhouse condition. ${ }^{\mathrm{b}} \mathrm{LOD}=$ logarithm of odds. ${ }^{\mathrm{c}} \mathrm{R}^{2}=$ the percentage of phenotypic variation the QTL explained.

QYrPI181410.wgp-4AS was detected by SNP IWB9590 with the logarithm of odds (LOD) of 3.3 and 2.7 for PSTv-4 and PSTv-14, respectively (Table 4). It peaked at the genetic location of $43.0 \mathrm{cM}$ and spanned a relatively small interval of $4.0 \mathrm{cM}$ on the short arm of chromosome $4 \mathrm{~A}$. This QTL explained $11.2 \%$ and $5.4 \%$ of the phenotypic variation to PSTv- 4 and PSTv-14, respectively.

QYrPI181410.wgp-4BL was detected by IWB6961 on 4BL using two sets of IT data with both PSTv-4 and PSTv-14 with the LOD ranging from 2.5 to 3.6 (Table 4). It peaked at the location of $71.0 \mathrm{cM}$ and spanned an interval of $20.0 \mathrm{cM}$ region. This QTL explained $8.6 \%$ and $11.0 \%$ of the phenotypic variation to PSTv-4 and PSTv-14, respectively. QYrPI181410.wgp-5BL.1 was detected by IWB865 and peaked at the location of $211.0 \mathrm{cM}$ with an interval of $10.0 \mathrm{cM}$ on 5BL. It explained $15.1 \%$ and $14.0 \%$ of the phenotypic variation to PSTv-4 and PSTv-14, respectively.

\subsection{QTL for HTAP Resistance}

Using 12 sets of phenotypic data from field tests and two sets of adult-plant phenotypic data from the greenhouse tests (Table S1), we identified a total of six resistance QTL including QYrPI181410.wgp-1BL, QYrAvS.wgp-2BS, QYrAvS.wgp-2BL, QYrPI181410.wgp-4BL, QYrPI181410.wgp-5AS, and QYrPI181410.wgp-5BL.2 (Table 5, Figure 3).

Table 5. Quantitative trait loci (QTL) associated with high temperature adult-plant resistance (HTAP) to stripe rust detected in wheat genotype PI 181410 and AvS using the recombinant inbred line population derived from AvS $\times$ PI 181410 .

\begin{tabular}{|c|c|c|c|c|c|c|c|c|}
\hline QTL & $\begin{array}{l}\text { Phenotypic } \\
\text { Data Set }^{\text {a }}\end{array}$ & $\begin{array}{l}\text { Flanking } \\
\text { Markers }\end{array}$ & $\begin{array}{c}\text { Peak } \\
\text { Marker }\end{array}$ & $\begin{array}{c}\text { Peak } \\
\text { Position }\end{array}$ & LOD $^{b}$ & $\begin{array}{l}\text { Interval of } \\
\text { QTL (cM) }\end{array}$ & $R^{2}(\%)^{c}$ & $\begin{array}{l}\text { Additive } \\
\text { Effect } d\end{array}$ \\
\hline \multirow[t]{5}{*}{ QYrPI181410.wgp-1BL } & MV15-IT & $\begin{array}{l}\text { IWB6507, } \\
\text { IWB72918 }\end{array}$ & IWB6507 & 160 & 6.4 & $159.0-165.0$ & $10.5^{* * *}$ & 1.0 \\
\hline & MV16-IT & $\begin{array}{l}\text { IWB3715, } \\
\text { IWB9772 }\end{array}$ & IWB6507 & 158 & 2.9 & $154.0-173.0$ & $14.2^{* * *}$ & 1.0 \\
\hline & MV15-rAUDPC & $\begin{array}{l}\text { IWB6507, } \\
\text { IWB73714 }\end{array}$ & IWB6507 & 160 & 3.6 & $159.0-168.0$ & $7.3^{* * *}$ & 7.8 \\
\hline & MV17-IT & $\begin{array}{l}\text { IWB6507, } \\
\text { IWB72918 }\end{array}$ & IWB6507 & 160 & 4.5 & $159.0-166.0$ & $10.6^{* * *}$ & 0.9 \\
\hline & Pu16-IT & $\begin{array}{l}\text { IWB3715, } \\
\text { IWB25646 }\end{array}$ & IWB13472 & 157 & 4.2 & $154.0-162.0$ & $5.8^{* * *}$ & 0.5 \\
\hline \multirow[t]{2}{*}{ QYrAvS.wgp-2BS } & MV15-IT & $\begin{array}{l}\text { IWB13249, } \\
\text { IWB11101 }\end{array}$ & IWB11101 & 18.0 & 3.1 & $12.0-20.0$ & $5.6^{* * *}$ & -0.4 \\
\hline & MV16-IT & $\begin{array}{l}\text { IWB13249, } \\
\text { IWB11101 }\end{array}$ & IWB11101 & 18.0 & 3.0 & $12.0-20.0$ & $5.0^{* * *}$ & -0.5 \\
\hline$Q Y r A v S . w g p-2 B L$ & Pu16-IT & $\begin{array}{l}\text { IWB51607, } \\
\text { IWB47487 }\end{array}$ & IWB10669 & 114.0 & 4.0 & 109.0-118.0 & $7.2 * * *$ & -0.5 \\
\hline
\end{tabular}


Table 5. Cont.

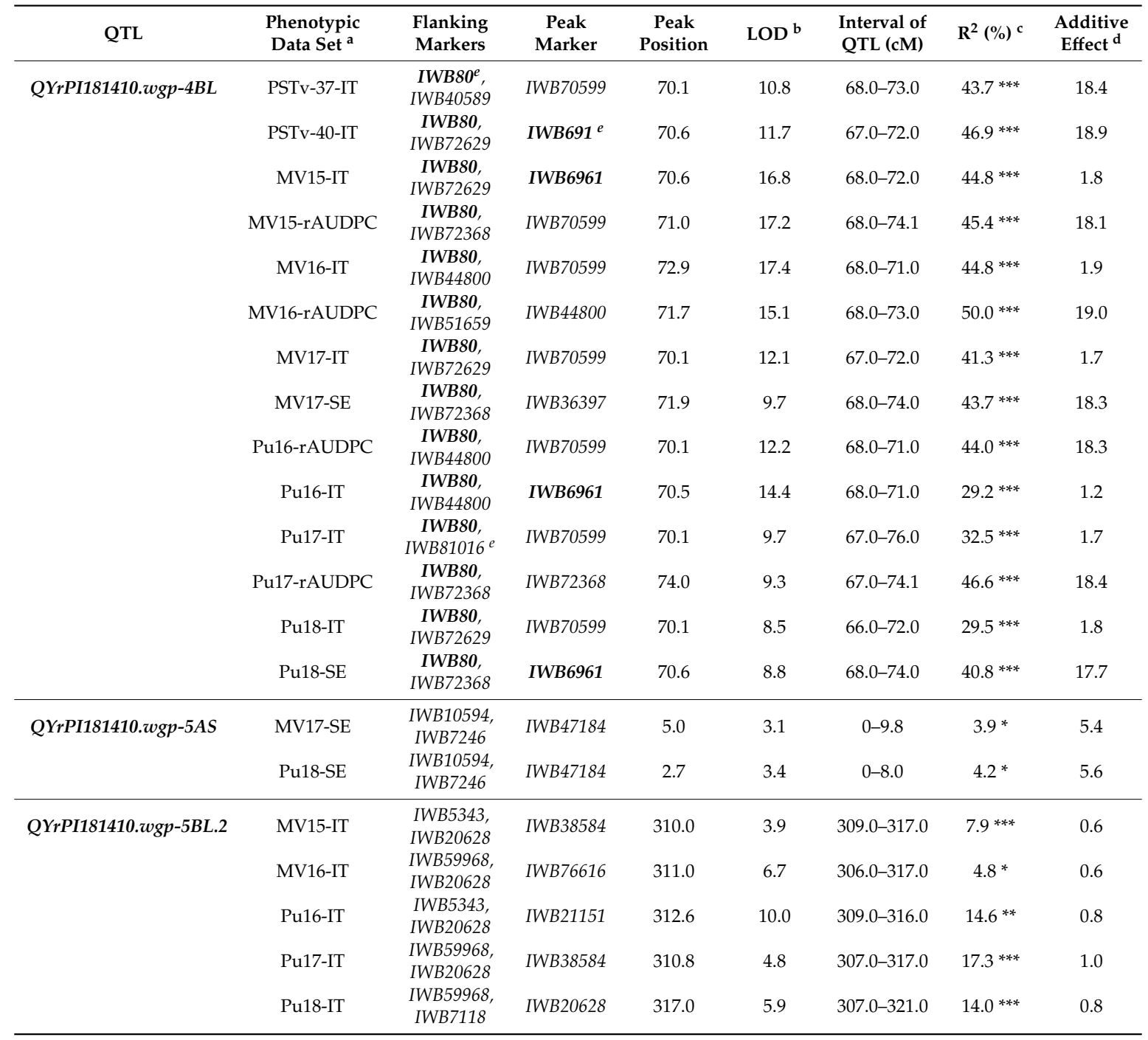

${ }^{a}$ In the phenotypic data sets, MV = Mount Vernon and Pu = Pullman; $15=2015,16=2016$, and 17 = 2017; IT $=$ infection type, rAUDPC $=$ relative area under the disease progress curve, and SE $=$ severity. ${ }^{b}$ LOD $=$ logarithm of odds. ${ }^{c} \mathrm{R}^{2}=$ the percentage of phenotypic variation the QTL explained. ${ }^{\mathrm{d}}$ additive effect: the negative value indicates that the resistance allele is from AvS (Avocet S). ${ }^{e}$ The SNP markers highlighted in bold were successfully converted to KASP markers.

QYrPI181410.wgp-1BL was detected by IWB6507 using four sets of field IT data at Mount Vernon in 2015, 2016, and 2017 and at Pullman in 2016, and one set of rAUDPC data from Mount Vernon in 2015 . It explained $6.6 \%$ to $10.9 \%$ of the phenotypic variation (Table 5). The overlap interval of QYrPI181410.wgp-1BL spanned about 6.0 cM genomic region on 1BL with LOD ranging from 2.9 to 6.4 (Table 5).

A major QTL, QYrPI181410.wgp-4BL, was consistently detected using all 12 sets of phenotypic data from field tests and two sets of data from greenhouse tests. The LOD value of QYrPI181410.wgp- $4 B L$ ranged from 8.5 to 17.4 (Table 5, Figure 4). It peaked around the genetic position of $70.1 \mathrm{cM}$ by SNP markers IWB70599 and IWB6961 within an interval of $3.0 \mathrm{cM}(68.0 \mathrm{cM}$ to $71.0 \mathrm{cM})$ on $4 \mathrm{BL}$ and explained $29.2 \%$ to $50.0 \%$ of the phenotypic variation. However, the phenotypic effect of QYrPI181410.wgp- $4 B L$ was lower (29.2 to 32.5) for infection type than for severity in Pullman, WA. 


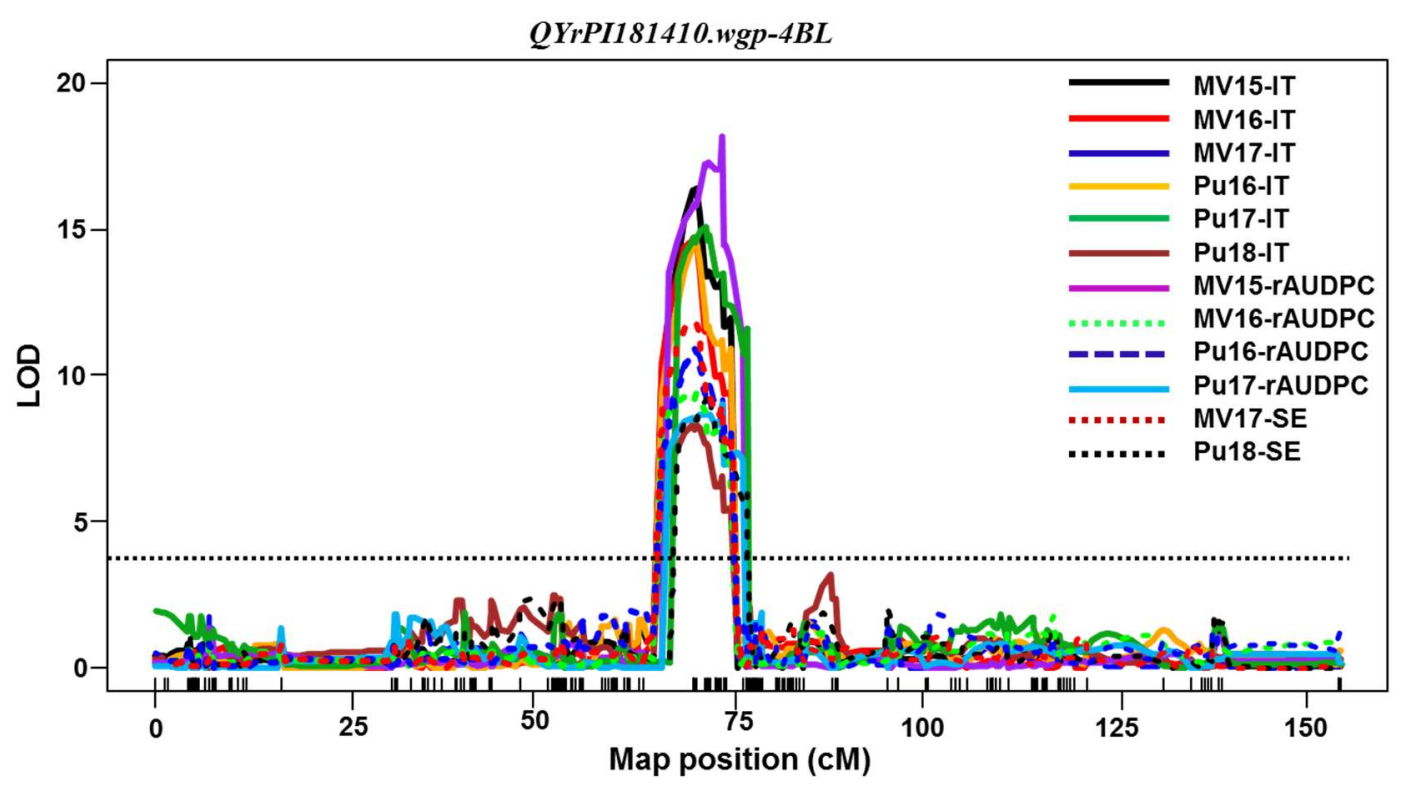

Figure 4. The LOD curves of QYrPI181410.wgp-4BL detected on chromosome 4B using 12 sets of data collected from the field tests across all environments including six sets of infection type (IT) data, four sets of relative area under the disease progress curve (rAUDPC) data, and two sets of disease severity (SE) data. The horizontal dotted line indicates the LOD threshold at 3.0.

Two minor QTL, QYrAvS.wgp-2BS and QYrAvS.wgp-2BL, were detected on chromosome 2B and the effects were provided by the susceptible parent AvS (Table 5, Figure 3). QYrAvS.wgp-2BS was detected with two sets of IT data from Mount Vernon and explained 5.0\% 5.6\% of IT variation. QYrAvS.wgp-2BL was only detected with one set of IT data from the Pullman location and explained $7.2 \%$ of IT variation. Both QTL did not have any significant effect on rAUDPC.

QYrPI181410.wgp-5AS was identified with two sets of SE data as a minor QTL conferring resistance on reducing severity of stripe rust in PI 181410 (Table 5). This QTL was detected by IWB47184 and located on the telomeric end of $5 \mathrm{AS}$. It explained $3.9 \%$ to $4.2 \%$ of SE variation.

QYrPI181410.wgp-5BL.2 was detected with five sets of field IT data from Mount Vernon in 2015 and 2016 and Pullman in 2016, 2017, and 2018 (Table 5, Figure 3). It was located within a $7.0 \mathrm{cM}$ genomic interval and peaked around $310.0 \mathrm{cM}$ close to the end of 5BL. This QTL explained $4.8 \%$ and $7.9 \%$ of the IT variation of data from Mount Vernon. However, it explained $14.6 \%$ to $17.3 \%$ of IT variation of data from Pullman, WA. This result suggested that the effect of QYrPI181410.wgp-5BL.2 varies among the environments.

\subsection{Effects of QTL Combinations}

To estimate the effects of QTL combinations, the RILs were classified into different groups containing different QTL combinations based on the genotypes spanning the QTL intervals. According to the ASR QTL identified in the greenhouse seedling tests, RILs were classified into four groups (Figure 5). Although these QTL contributed minor effects to reduce the stripe rust to specific races including PSTv-4 and PSTv-14 in the greenhouse conditions, the RILs containing more QTL showed higher resistance than those containing few QTL. 


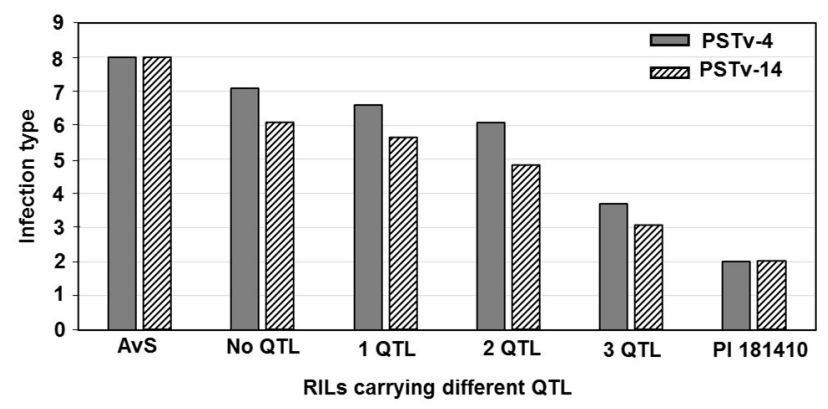

Figure 5. Comparison of mean infection type values of recombinant inbred lines (RILs) derived from cross AvS $\times$ PI 181410 containing different all-stage resistance QTL combination.

The similar trends were also found for HTAP resistance. Four QTL for HTAP resistance were identified in PI 181410. Based on the markers for these QTL, RILs were classified into five groups (Figure 6). QYrPI181410.wgp-4BL had a higher effect than QYrPI181410.wgp-1BL, QYrPI181410.wgp-5AS, and QYrPI181410.wgp-5BL.2. The resistance level was greatly increased when the major QTL QYrPI181410.wgp-4BL was in combination with minor QTL QYrPI181410.wgp-1BL and/or QYrPI181410.wgp-5BL.2. The RILs containing one of the QTL showed a lower IT and rAUDPC than those without any QTL. The RILs containing more resistance QTL had a higher level of resistance (lower IT and/or rAUDPC values) than those containing a smaller number of QTL (Figure 6A,B). These results indicated that the combinations of different QTL increased the level of resistance through additive interactions.
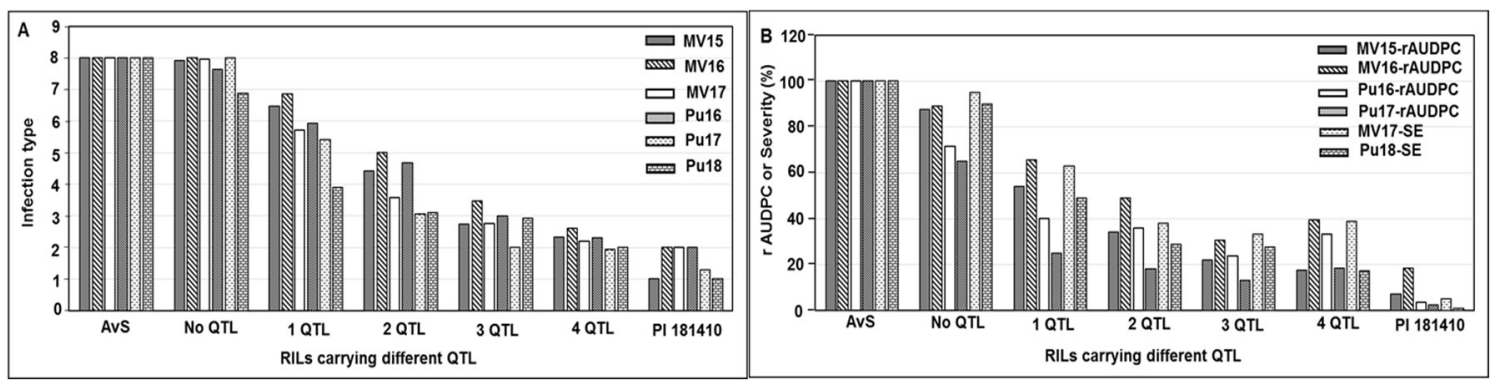

Figure 6. Comparison of mean infection type (A) and rAUDPC/severity (SE) (B) values of recombinant inbred lines (RILs) derived from cross AvS $\times$ PI 181410 containing different HTAP resistance QTL combination.

\subsection{Kompetitive Allele Specific PCR (KASP) Markers for the Major-Effect QTL}

As QYrPI181410.wgp-4BL was the major-effect QTL for HTAP resistance identified in PI 181410 and detected in all greenhouse and field tests, KASP markers were developed for this QTL. Five KASP markers were successfully converted from their original SNP markers (Table 6). Three of five KASP markers, KIWB81016, KIWB6961, and KIWB80, which were developed from SNP markers IWB81016, IWB6961, and IWB80, respectively, were confirmed to be polymorphic between PI 181410 and AvS. When tested on the RIL population, the KASP markers produced the same genotypes as their original SNP markers among the individual RILs.

The three polymorphic KASP markers, were used to determine their polymorphisms in 155 wheat cultivars and breeding lines from the US (Table S3). These markers had polymorphism rates $45.8 \%$, $69.0 \%$, and $100 \%$, respectively. As expected, none of the 155 wheat accessions had the same marker haplotype of PI 181410 for the three KASP markers. This result indicated that QYrPI181410.wgp-4BL was absent in the collection of US wheat cultivars and breeding lines, and the KASP markers in combination, or individual marker KIWB80, should be useful in MAS for incorporating this QTL into new wheat cultivars. 
Table 6. Primer sequences of KASP markers for detecting the major stripe rust resistance QTL, QYrPI181410.wgp-4BL, from PI 181410 among 158 wheat cultivars or breeding lines.

\begin{tabular}{|c|c|c|}
\hline SNP ID (90K) & KASP Primer & Sequences $\left(5^{\prime}-3^{\prime}\right)$ \\
\hline \multirow[t]{3}{*}{ IWB81016 } & IWA7752A & GAAGGTGACCAAGTTCATGCTCCTATACAACAGAACACTGGGAACA \\
\hline & IWA7752B & GAAGGTCGGAGTCAACGGATTCCTATACAACAGAACACTGGGAACG \\
\hline & IWA7752C & AGAGTCGCCACGATCGTTTAACT \\
\hline \multirow[t]{3}{*}{ IWB26339 } & IWB26339A & GAAGGTGACCAAGTTCATGCTAGTTCTGGGTCGTCATTTTGT \\
\hline & IWB26339B & GAAGGTCGGAGTCAACGGATTAGTTCTGGGTCGTCATTTTGC \\
\hline & IWB26339C & ATGATTTGTGTTGTAGGGGGT \\
\hline \multirow[t]{3}{*}{ IWB44800 } & IWB44800A & GAAGGTGACCAAGTTCATGCTCCTCACAATGTTATGATTGACCAT \\
\hline & IWB44800B & GAAGGTCGGAGTCAACGGATTCCTCACAATGTTATGATTGACCAC \\
\hline & IWB44800C & CCTCGAAGCAACACGAACG \\
\hline \multirow[t]{3}{*}{ IWB6961 } & IWB6961A & GAAGGTGACCAAGTTCATGCTTGTTTGCTCTCATGAACGCTT \\
\hline & IWB6961B & GAAGGTCGGAGTCAACGGATTTGTTTGCTCTCATGAACGCTC \\
\hline & IWB6961C & CAGTTTCACATTCAACAGAAAGC \\
\hline \multirow[t]{3}{*}{ IWB80 } & IWB80A & GAAGGTGACCAAGTTCATGCTAAGAGGCCATGCTTCCCTA \\
\hline & IWB80B & GAAGGTCGGAGTCAACGGATTAAGAGGCCATGCTTCCСТC \\
\hline & IWB80C & AAGTTCAAGCATGGCAAGCA \\
\hline
\end{tabular}

\section{Discussion}

Wheat landrace PI 181410 has been consistently showing high-level resistance to stripe rust in the US Pacific Northwest region since 2006. The four-way tests conducted with predominant and most virulent races of Pst in both seedling and adult-plant stages at both LT and HT profiles showed that PI 181410 has both race-specific ASR and high-level HTAP resistance. Using the high-density genetic maps and multiple environmental tests for QTL mapping, we identified three QTL in PI 181410 for ASR, QYrPI181410.wgp-4AS, QYrPI181410.wgp-4BL, and QYrPI181410.wgp-5BL.1. In addition to the QTL on 4AS, we identified three more QTL, designated as QYrPI181410.wgp-1BL, QYrPI181410.wgp-5AS, and QYrPI181410.wgp-5BL.2 in PI 181410 conferring resistance observed at the adult-plant stage in the fields. Interestingly, we also detected two QTL, designated as QYrAvS.wgp-2BS and QYrAvS.wgp-2BL, from AvS with minor effects only on IT at Mount Vernon and Pullman, respectively. Each of the QTL were located within a relatively small genomic region.

QYrPI181410.wgp-1BL associated with minor HTAP resistance was detected at the distal end of the long arm of 1B. Yr29 is also located at the distal end of 1BL [13]. At the Yr29 location, eleven QTL were identified with different phenotypic effects [14]. Six of the eleven QTL have been reported to provide significant effects on stripe rust resistance in multiple environmental tests, such as QYr.sun-1B, QYr.jic-1B, QYr.tam-1B, QYr.cim-1BL, QYr-1B, QYr.cim-1BL, and QYr.1BL [15-20]. Other QTL including QYrPst.jic-1B were reported to have minor effects on stripe rust resistance [14,21]. Based on the chromosome regions, QYrPI181410.wgp-1BL is likely Yr29. The present study and previous studies suggests that the long arm of chromosome $1 \mathrm{~B}$ carries multiple genes for resistance to stripe rust.

Two minor-effect QTL, QYrAvS.wgp-2BS and QYrAvS.wgp-2BL, were identified from susceptible parent AvS. QYrPI181410.wgp-2BS was mapped close to the distal end of the short arm. According to previous studies, two QTL including QYr.inra-2BS [22] and QYr.wgp-2B.1 [23] were mapped at the same region as QYrAvS.wgp-2BS. QYr.inra-2BS contributed moderate resistance to stripe rust at both seedling and adult-plant stages [22]. Identified in several US Pacific Northwest wheat cultivars by genome-wide association mapping, QYr.wgp-2B.1 contributed adult-plant resistance to stripe rust and was significantly associated with both IT and SE in several environments including Pullman and Mount Vernon, Washington [23]. However, QYrPI181410.wgp-2BS was only associated with IT in Mount Vernon. Thus, QYrAvS.wgp-2BS is likely a locus different from QYr.inra-2BS and QYr.wgp-2B.1. QYrAvS.wgp-2BL was only detected using one set of IT data in a single field condition in Pullman. It was located at the distal end of 2BL. Rosewarne et al. [17] reported a minor QTL affecting adult-plant resistance in AvS flanked by simple sequence repeat (SSR) markers Xgwm1027 and Xgwm619 at the same location on chromosome 2BL as QYrAvS.wgp-2BL. Since their QTL and QYrAvS.wgp-2BL were detected in the same wheat genotype in the same chromosomal location, they should be the same gene. 
QYrPI181410.wgp-5AS had a minor effect on reducing disease severity in PI 181410 and was located at the telomere of chromosome 5AS. As no stripe rust resistance QTL have been reported at this location, the $5 A S$ QTL is a new QTL for resistance to stripe rust.

Two QTL, QYrPI181410.wgp-5BL.1 and QYrPI181410.wgp-5BL.2, from PI 181410 were mapped to different locations on chromosome 5BL. These QTL conferred resistance to stripe rust at different plant growth stages. QYrPI181410.wgp-5BL.1, associated with ASR, was located at the middle region of 5BL, while QYrPI181410.wgp-5BL.2, associated with HTAP resistance, was mapped to the distal end of 5BL. Five previously reported QTL, including QYr.caas-5BL.1 from Libellula [24], QYrdr.wgp-5BL.2 [25], QYrco.wpg-5B [26], QYr.jirc-5BL [27], and QYr.inra-5BL.1, were at the chromosomal regions similar to QYrPI181410.wgp-5BL.1 based on their flanking markers. All these QTL exhibit adult-plant resistance to multiple races, however, QYrPI181410.wgp-5BL.1 conferred ASR to races PSTv-4 and PSTv-14. Based on the different types of resistance, QYrPI181410.wgp-5BL.1 may be different from those known QTL, but further work is needed to determine their genetic relationships. Two QTL, QYr.caas-5BL.2 flanked by Xbarc142 and Xgwm604 [24] and QYr.caas- 5BL.3 flanked by SSR markers Xwmc75 and Xbarc275 [28], were mapped to the distal end of 5BL [14]. Compared with the consensus map [29], QYrPI181410.wgp-5BL.2 should be close to the far distal end of the 5BL and not in the same region as the above two QTL. Thus, QYrPI181410.wgp-5BL.2 is likely a new QTL for stripe rust resistance.

Among the QTL identified in PI 181410, QYrPI181410.wgp-4BL was the most effective. It was consistently detected using all 16 sets of phenotypic data collected from all field tests and both seedling and adult-plant tests in the greenhouse. Its effect was much stronger in the adult-plant stage in the fields and at $\mathrm{HT}$ in the greenhouse test than the seedling stage at LT, and therefore, it should be considered as a HTAP resistance gene. QYrPI181410.wgp-4BL was mapped to an interval of $9.0 \mathrm{cM}(67.0 \sim 76.0 \mathrm{cM})$ close to the centromeric region of $4 \mathrm{BL}$ according to the consensus map $[29,30]$. The overlap of the genomic regions detected by all 16 data sets narrowed the QTL to $4.0 \mathrm{cM}$ between 68.0 and $72.0 \mathrm{cM}$ positions (Table 5, Figure 4). In previous studies, $Y r 50$ and $Y r 62$ were also mapped to the same location on 4BL as QYrPI181410.wgp-4BL. The dominant resistance gene, Yr50, derived from Thinopyrum intermedium, was mapped on 4BL and flanked by SSR markers Xbarc1096 and Xwmc47, and Yr50 exhibited ASR to all tested Chinese $P$ st races and eight US predominant $P_{s t}$ races including PSTv-40 [31,32]. In contrast, QYrPI181410.wgp- $4 B L$ is from common wheat landrace PI 181410 and confers strong HTAP resistance with only a minor effect at seedling stage. The differences clearly indicate that QYrPI181410.wg $p$ - $4 B L$ is different from Yr50. Yr62, from Portuguese spring wheat variety PI 192252, was flanked by SSR markers Xgwm192 and Xgwm251 on 4BL [32]. This gene provides a higher level HTAP resistance (40\%-60\% of phenotypic variation) than QYrPI181410.wgp-4BL (29.2\%-46.9\%). Yr62 is not effective at the seedling stage. Thus, QYrPI181410.wg $p$-4BL is likely different from Yr62. Further studies are needed to determine their relationship using the allelism test approach.

Two major QTL, QPst.jic- $4 B$ and QYrva.vt-4BL, were also mapped around the centromeric region on 4BL $[21,23]$. QPst.jic-4B, identified from a German winter wheat cultivar Alcedo, was flanked by amplified fragment length polymorphism (AFLP) marker S24M37_130 and SSR marker Xcfd39 and peaked at Xwmc692 on 4BL [21]. QYrva.vt-4BL, identified from a soft red winter wheat VA00W-38, was flanked by SSR markers Xwmc692 and Xgwm149 on 4BL [33]. Both QPst.jic-4B and QYrva.vt-4BL explained less effect (17.9\%-28.9\% and $19.3 \%$, respectively) of the phenotypic variation. Thus, QYrPI181410.wgp-4BL is unlikely the same as any of these two QTL in winter wheat cultivars. As QYrPI181410.wgp-4BL is the most useful among the QTL detected in the present study, we developed three KASP markers for this QTL. The KASP markers performed in the same way as the original SNP markers between the parents and among the RILs, and they were highly polymorphic when tested with 155 wheat cultivars and breeding lines from the US Pacific Northwest. None of the wheat materials have the KIWB80 allele or the PI 181410 haplotype of the three markers. Therefore, the KASP marker KIWB80 or the combination of the three markers can be used in MAS for incorporating the effective QTL into various wheat backgrounds for developing new cultivars with durable, high-level resistance to stripe rust. 


\section{Materials and Methods}

\subsection{Plant Materials}

PI 181410, an Afghanistan spring wheat landrace with resistance to stripe rust, was used as the male parent in crossing with Avocet S (AvS), a stripe rust susceptible Australian spring wheat genotype, as a female parent. A recombinant inbred line (RIL) population of the cross was developed using the single-seed decent method. A total of 170 RILs of the $F_{5}, F_{6}, F_{7}$, and $F_{8}$ generations were used for phenotyping the response to stripe rust in different years and locations, and the first $114 \mathrm{~F}_{7}$ RILs of the $\mathrm{F}_{7}$ generation were used for SNP genotyping. A collection of 155 US Pacific Northwest spring and winter wheat cultivars and breeding lines was used to determine polymorphisms of the KASP markers developed in this study.

\subsection{Evaluation of PI 181410 and RILs for Stripe Rust Reaction in the Greenhouse}

To characterize the stripe rust resistance in PI 181410, seedlings and adult plants were tested with five US predominant or most virulent races of Pst, PSTv-4 (isolate 2013-445), PSTv-14 (isolate 2012-116), PSTv-37 (isolate 2012-114), PSTv-40 (isolate 2009-78), and PSTv-51 (isolate 2011-366) [9,10], following the procedures as previously described [7,34]. Briefly, about 10-15 seeds of each line were planted in one pot in a plastic tray $(53.43 \times 27.5 \times 6.10 \mathrm{~cm}$, containing 72 pots $)$ filled with soil mixture. Seedlings at two-leaf stage [growth stage (GS) 10] [35] were inoculated with urediniospores of a selected race mixed with talc at the ratio of 1:20. The inoculated plants were incubated in a dew chamber at $10{ }^{\circ} \mathrm{C}$ in darkness for $24 \mathrm{~h}$ for urediniospores to germinate and infect the plants, and then transferred to a growth chamber at a diurnal cycle of low-temperature (LT) gradually changing from $4{ }^{\circ} \mathrm{C}$ at 2:00 a.m. to $20^{\circ} \mathrm{C}$ at 2:00 p.m. with a $16 \mathrm{~h}$ light $/ 8 \mathrm{~h}$ dark photoperiod. For high-temperature (HT) tests, after incubating in the dew chamber for $24 \mathrm{~h}$ at $10^{\circ} \mathrm{C}$ in darkness, the inoculated plants were placed in a growth chamber at a diurnal temperature cycle gradually changing from $10{ }^{\circ} \mathrm{C}$ at $2: 00$ a.m. to $30{ }^{\circ} \mathrm{C}$ at 2:00 p.m. with the same photoperiod cycle. For adult-plant tests at both LT and HT profiles, 3 plants grown in a pot $(15 \times 15 \times 18 \mathrm{~cm})$ to the boot stage (GS 45) were inoculated, incubated, and grown under the same conditions as described above. For each race-temperature test, a total of 9 plants in 3 pots were evaluated. Stripe rust infection type (IT) of each plant was scored at 18 to 21 days after inoculation using the 0 to 9 scales [36]. For the seedling tests, ITs of the second leaves were recorded while those of the flag leaves were recorded for the adult-plant tests. Seedlings or adult plants of AvS were included as the susceptible check in each stage-temperature test.

Races PSTv-4 and PSTv-14 of Pst were used for testing the $170 \mathrm{~F}_{7}$ RILs at the seedling stage at the LT profile $\left(4-20^{\circ} \mathrm{C}\right)$ with three biological replicates based on their relative avirulence on the seedlings of PI 181410. In these tests, PI 181410 and AvS were included as the resistant and susceptible checks, respectively, and the $18 Y r$ single-gene differentials [9] were included to ensure the correct race. PSTv-37 and PSTv-40 were used to test the adult plants of 170 RILs at the HT profile $\left(10-30{ }^{\circ} \mathrm{C}\right)$ based on their virulence on seedlings at LT and avirulence on adult plants of PI 181410 at HT. PI 181410 and AvS were also included as the resistant and susceptible checks, respectively. The inoculation, incubation, growth of plants, and IT data record were the same as described above for the seedling LT and adult plant HT tests for the parental lines.

\subsection{Evaluation for Stripe Rust Responses of PI 181410 and RILs in the Fields}

The stripe rust responses of PI 181410, together with other wheat genotypes, were tested under natural Pst infections in field nurseries at Mount Vernon in northwestern Washington and Pullman in southeastern Washington since 2006. The two locations are about $500 \mathrm{~km}$ apart with different climate conditions and different Pst races $[9,10]$. The $170 \mathrm{~F}_{5}, \mathrm{~F}_{6}, \mathrm{~F}_{7}$, or $\mathrm{F}_{8}$ RILs were consecutively phenotyped in both locations in 2015, 2016, 2017, and 2018, respectively. The parental lines and RILs were planted in a randomized block design with three replications. About 20-30 seeds of each line were planted in a $50 \mathrm{~cm}$ row with $20 \mathrm{~cm}$ between rows. As a susceptible check and spreader, AvS was planted every 
20 rows and around the field. Stripe rust IT and severity (SE) were recorded three times at the tillering stage (GS 30-31), booting stage (GS 45), and flowering to grain-filling stages (GS 60-70). Severity was recorded as the percentage of leaf area infected. The SE data were used to calculate the area under the disease progress curve (AUDPC) [37], which was then converted to relative AUDPC (rAUDPC) as percentage of the mean AUDPAC (as 100\%) of susceptible parent AvS.

\subsection{Statistical Analyses of the Phenotypic Data}

Broad-sense heritability $\left(H^{2}\right)$ of phenotypic traits was calculated using the heritability package of R program following the formula $H^{2}=\delta^{2}(\mathrm{G}) /\left[\delta^{2}(\mathrm{G})+\delta^{2}\left(\mathrm{G}^{*} \mathrm{~L}\right) / l+\delta^{2}\left(\mathrm{G}^{*} \mathrm{Y}\right) / y+\delta^{2}(\mathrm{E}) / \mathrm{ly}\right]$, where $\delta^{2}(\mathrm{G})=$ genotypic variance, $\delta^{2}\left(\mathrm{G}^{*} \mathrm{~L}\right)=$ variance of genotype by location interaction, $\delta^{2}\left(\mathrm{G}^{*} \mathrm{Y}\right)=$ variance of genotype by year interaction, $\delta^{2}(\mathrm{E})=$ residual variance, $l=$ number of location, and $y=$ number of years (https://cran.r-project.org/web/packages/heritability/heritability.pdf).

The analysis of variance (ANOVA) was performed for the IT data, SE, and rAUDPC using the aov function of the R program (version 3.5.2, https://www.r-project.org) to determine the effects of genetic and environmental factors and their interactions. Correlation coefficients were calculated using the correlation function in Excel for rAUDPC and IT data set to compare population responses across the four field environments and two greenhouse tests.

\subsection{Genotyping the Parental Lines and RILs}

The parental lines and $F_{7}$ RILs were planted in the greenhouse for DNA extraction. Genomic DNA was extracted from the leaves harvested from a single plant of each $\mathrm{F}_{7}$ RIL using the CTAB (cetyl trimethylammonium bromide) method [38]. DNA quality was determined using $0.8 \%$ agarose gel electrophoresis, and DNA concentration was measured using a NanoDrop ND-1000 (Thermo Scientific, Wilmington, DE, USA). Each DNA sample was diluted to $50 \mathrm{ng} / \mu \mathrm{L}$ for use in genotyping. Out of the $170 \mathrm{~F}_{7}$ RILs, the first 114 RILs and parental lines were genotyped using the 90K Illumina ${ }^{\circledR}$ iSelect wheat SNP assay [39] at the USDA-ARS Biosciences Research Laboratory, Fargo, ND. The reduced number of RILs genotyped was due to a budget limitation. SNP calling and clustering were determined using the functions of software GenomeStudio v2011.1 (Illumina, San Diego, CA, USA) based on the described genotypic data. Monomorphic SNPs, SNPs with more than 10\% missing data, and RILs with 30\% missing data were removed before further analyses.

\subsection{Genetic Map Construction and QTL Mapping}

SNP markers polymorphic between the parents were tested for the goodness of the fit to the 1:1 segregation ratio among the RILs using the chi-squared test in JoinMap 4.1 (https://www.kyazma.nl/ index.php/JoinMap/Manual/). SNP markers that did not fit the 1:1 ratio $(p<0.01)$ were excluded for further analyses.

A genetic map was constructed using software JoinMap 4.1 [40] with the Kosambi mapping function [41]. The threshold value of logarithm of odds (LOD) score of 3.0 was applied to select the genetic linkage groups. The corresponding chromosome of each linkage group was determined based on the chromosome locations of SNPs from the 90K array [39]. The graphic genetic maps were generated using software MapChart v2.3 [42].

The IT data of both seedling stage and adult-plant stage in each year-location environment were used directly for QTL analysis. rAUDPC data were used for QTL mapping if the test had three sets of SE data. If only one set of SE data in the adult-plant stage was collected, as in the case of 2018 Pullman location, the SE data were also directly used for QTL mapping. QTL analysis was performed using both single-marker analysis (SMA) and composite interval mapping (CIM) with the Haley-Knott regression (HK) method in the R/qtl package of RStudio version 1.0.143 [43]. For QTL mapping, the probabilities of association between the genotypes and phenotypes were calculated using the function calc.genoprob with a step size of $1 \mathrm{cM}$ distance and a genotype error of 0.01. SMA was performed using the scanone function and followed by CIM using the cim function. For each set of phenotypic data, a permutation 
test was performed using the n.perm function $(n=1000)$ to obtain a genome-wide LOD significance threshold. The resistance QTL interval with $95 \%$ confidence was obtained using the bayesint function. SNP markers closest to the QTL peak were identified using find.marker. The effect of each QTL was estimated using the plotPXG function. For the phenotypes with more than one QTL identified, the effect of each QTL was estimated using the multiple QTL mapping method in the R/qtl package [44]. The additive effect for each QTL was estimated using the get.ests = TRUE function. The interaction between two QTL was calculated using the function addint in the multiple QTL mapping method.

\subsection{Developing KASP Markers}

SNP markers flanking or at the peaks of the identified resistance QTL were selected for developing KASP markers using the information provided by PolyMarker (http://polymarker.tgac.ac.uk). Primers of KASP markers were synthesized by Sigma-Aldrich Inc. (Saint Louis, MO, USA). The KASP markers were validated using the parental lines PI 181410 and AvS and the RILs. The PCR reaction, amplification, and detection for KASP markers were conducted following the previously described method [45]. The PCR assay was performed in a LightCycler ${ }^{\circledR} 480$ System (Roche Applied Science, Indianapolis, IN, USA) using the endpoint genotyping analysis. To determine the polymorphisms of the KASP markers in various wheat backgrounds, 155 spring and winter wheat cultivars and breeding lines from the US Pacific Northwest were tested with the markers for the major effective QTL.

\section{Conclusions}

This study determined that the spring wheat landrace PI 181410, originally from Afghanistan, possesses both race-specific ASR and nonrace-specific HTAP resistance to stripe rust. Three QTL conferring ASR in PI 181410 were mapped to chromosomes 4BS, 4BL, and 5BL; and four QTL (including the 4BL QTL detected in the seedling stage) conferring HTAP resistance were mapped on chromosomes 1BL, 4BL, 5AS, and 5BL. The 1BL QTL was identified as $Y r 29$, and the four other QTL in PI 181410 are potentially new. Two QTL were mapped on 2BS and 2BL from the susceptible parent AvS with minor effects on resistance in the field. The 2BL QTL is the same as a previously mapped QTL in the same variety, while the 2BS QTL is a potentially new locus. As the 4BL QTL was the most effective among the identified QTL and potentially new, three KASP markers (KIWB81016, KIWB6961, and KIWB80) were developed from its associated SNPs. These markers, especially KIWB80, were highly polymorphic among the US Pacific Northwest wheat cultivars and breeding lines. The resistant germplasm, identified QTL, and KASP markers for the most effective QTL are useful for developing wheat cultivars with durable, high-level resistance to stripe rust.

Supplementary Materials: Supplementary materials can be found at http://www.mdpi.com/1422-0067/21/2/478/s1.

Author Contributions: Y.L. conducted some of the greenhouse and field experiments, analyzed the phenotypic and genotypic data, constructed the linkage groups, mapped QTL, developed and validated the KASP markers, and drafted the manuscript. Y.Q. advanced the RIL population, conducted some of the greenhouse and field experiments, and extracted DNA for SNP genotyping. X.L. participated in some of the greenhouse and field experiments. M.W. advanced the progeny population to $\mathrm{F}_{5}$ and participated in the field and greenhouse experiments. X.C. conceived and directed the study, selected the resistant parent, made the cross, and wrote the manuscript. All authors have read and agreed to the published version of the manuscript.

Funding: This research was supported by the US Department of Agriculture, Agricultural Research Service (Project No. 2090-22000-018-00D), Vogel Foundation (Project No. 13Z-3061-6665), Washington Grain Commission (Project No. 13C-3061-5665; 13C-3061-3144), and Washington State University, Department of Plant Pathology, College of Agricultural, Human, and Natural Resource Sciences, Agricultural Research Center, HATCH Project Number WNP00461, Washington State University, Pullman, WA 99164-6430, USA.

Acknowledgments: The authors would like to thank Kent Evans and Jason Sprott for field preparation and weed control; and thank Shiaoman Chao for genotyping the wheat lines with 90K wheat SNP chip. The China Scholarship Council scholarship to Yanmin Qie is highly appreciated.

Conflicts of Interest: The authors declare no conflict of interest. The funders had no role in the design of the study; in the collection, analyses, or interpretation of data; in the writing of the manuscript, or in the decision to publish the results. 


\section{Abbreviations}

$\begin{array}{ll}\text { Pst } & \text { Puccinia striiformis f. sp. tritici } \\ \text { ASR } & \text { All-stage resistance } \\ \text { HTAP } & \text { High-temperature adult-plant } \\ \text { SNP } & \text { Single nucleotide polymorphism } \\ \text { MAS } & \text { Marker-assisted selection } \\ \text { IT } & \text { Infection type } \\ \text { SE } & \text { Severity } \\ \text { LT } & \text { Low temperature } \\ \text { HT } & \text { High temperature } \\ \text { RIL } & \text { Recombinant inbred line } \\ \text { ANOVA } & \text { Analysis of variance } \\ \text { rAUDPC } & \text { relative area under the disease progress curve } \\ \text { QTL } & \text { Quantitative trait locus or loci } \\ \text { SSR } & \text { Simple sequence repeat } \\ \text { AFLP } & \text { Amplified fragment length polymorphism } \\ \text { GS } & \text { Growth stage } \\ \text { SMA } & \text { Single marker analysis } \\ \text { CIM } & \text { Composite interval mapping } \\ \text { LOD } & \text { Logarithm of odd } \\ \text { KASP } & \text { Kompetitive allele specific PCR }\end{array}$

\section{References}

1. Gustafson, P.; Raskina, O.; Ma, X.F.; Nevo, E. Wheat evolution, domestication, and improvement. In Wheat Science and Trade; Carver, B.F., Ed.; Wiley-Blackwell: Ames, IA, USA, 2009; pp. 5-30.

2. Chen, X.M. Epidemiology and control of stripe rust [Puccinia striiformis f. sp. tritici] on wheat. Can. J. Plant Pathol. 2005, 27, 314-337. [CrossRef]

3. Wellings, C.R. Global status of stripe rust: A review of historical and current threats. Euphytica 2011, 179, 129-141. [CrossRef]

4. Savary, S.; Willocquet, L.; Pethybridge, S.J.; Esker, P.; McRoberts, N.; Nelson, A. The global burden of pathogens and pests on major food crops. Nat. Ecol. Evol. 2019, 3, 430-439. [CrossRef] [PubMed]

5. Chen, X.M. Integration of cultivar resistance and fungicide application for control of wheat stripe rust. Can. J. Plant Pathol. 2014, 36, 311-326. [CrossRef]

6. Line, R.F. Stripe rust of wheat and barley in North America: A retrospective historical review. Annu. Rev. Phytopathol. 2002, 40, 75-118. [CrossRef]

7. Chen, X.M. Review article: High-temperature adult-plant resistance, key for sustainable control of stripe rust. Am. J. Plant Sci. 2013, 4, 608-627. [CrossRef]

8. Sharma-Poudyal, D.; Chen, X.M.; Wan, A.M.; Zhan, G.M.; Kang, Z.S.; Cao, S.Q.; Jin, S.L.; Morgounov, A.; Akin, B.; Mert, Z.; et al. Virulence characterization of international collections of the wheat stripe rust pathogen, Puccinia striiformis f. sp. tritici. Plant Dis. 2013, 97, 379-386. [CrossRef]

9. Wan, A.M.; Chen, X.M. Virulence characterization of Puccinia striiformis f. sp. tritici using a new set of $Y r$ single-gene line differentials in the United States in 2010. Plant Dis. 2014, 98, 1534-1542. [CrossRef]

10. Wan, A.M.; Chen, X.M.; Yuen, J. Races of Puccinia striiformis f. sp. tritici in the United States in 2011 and 2012 and comparison with races in 2010. Plant Dis. 2016, 100, 966-975. [CrossRef]

11. Liu, L.; Wang, M.N.; Feng, J.Y.; See, D.R.; Chao, S.M.; Chen, X.M. Combination of all-stage and high-temperature adult-plant resistance QTL confers high level, durable resistance to stripe rust in winter wheat cultivar Madsen. Theor. Appl. Genet. 2018, 131, 1835-1849. [CrossRef]

12. Wang, M.N.; Chen, X.M.; Xu, L.S.; Cheng, P.; Bockelman, H.E. Registration of 70 common spring wheat germplasm lines resistant to stripe rust. J. Plant Regist. 2012, 6, 104-110. [CrossRef]

13. Lillemo, M.; Asalf, B.; Singh, R.P.; Huerta-Espino, J.; Chen, X.M.; He, Z.H.; Bjornstad, A. The adult plant rust resistance loci $L r 34 / Y r 18$ and $L r 46 / Y r 29$ are important determinants of partial resistance to powdery mildew in bread wheat line Saar. Theor. Appl. Genet. 2008, 116, 1155-1166. [CrossRef] [PubMed] 
14. Wang, M.N.; Chen, X.M. Stripe rust resistance. In Stripe Rust; Chen, X.M., Kang, Z.S., Eds.; Springer: Dordrecht, The Netherlands, 2017; pp. 383-444.

15. Melichar, J.P.E.; Berry, S.; Newell, C.; MacCormack, R.; Boyd, L.A. QTL identification and microphenotype characterisation of the developmentally regulated yellow rust resistance in the UK wheat cultivar Guardian. Theor. Appl. Genet. 2008, 117, 391-399. [CrossRef] [PubMed]

16. Zwart, R.S.; Thompson, J.P.; Milgate, A.W.; Bansal, U.K.; Williamson, P.M.; Raman, H.; Bariana, H.S. QTL mapping of multiple foliar disease and root-lesion nematode resistances in wheat. Mol. Breed. 2010, 26, 107-124. [CrossRef]

17. Rosewarne, G.M.; Singh, R.P.; Huerta-Espino, J.; Rebetzke, G.J. Quantitative trait loci for slow-rusting resistance in wheat to leaf rust and stripe rust identified with multi-environment analysis. Theor. Appl. Genet. 2008, 116, 1027-1034. [CrossRef]

18. Rosewarne, G.M.; Singh, R.P.; Huerta-Espino, J.; Herrera-Foessel, S.A.; Forrest, K.L.; Hayden, M.J.; Rebetzke, G.J. Analysis of leaf and stripe rust severities reveals pathotype changes and multiple minor QTLs associated with resistance in an Avocet $\times$ Pastor wheat population. Theor. Appl. Genet. 2012, 124, 1283-1294. [CrossRef]

19. Basnet, B.R.; Singh, R.P.; Ibrahim, A.M.H.; Herrera-Foessel, S.A.; Huerta-Espino, J.; Lan, C.X.; Rudd, J.C. Characterization of $Y r 54$ and other genes associated with adult plant resistance to yellow rust and leaf rust in common wheat Quaiu 3. Mol. Breed. 2014, 33, 385-399. [CrossRef]

20. Lan, C.X.; Rosewarne, G.M.; Singh, R.P.; Herrera-Foessel, S.A.; Huerta-Espino, J.; Basnet, B.R.; Zhang, Y.L.; Yang, E.N. QTL characterization of resistance to leaf rust and stripe rust in the spring wheat line Francolin \#1. Mol. Breed. 2014, 34, 789-803.

21. Jagger, L.J.; Newell, C.; Berry, S.T.; MacCormack, R.; Boyd, L.A. The genetic characterization of stripe rust resistance in the German wheat cultivar Alcedo. Theor. Appl. Genet. 2011, 122, 723-733. [CrossRef]

22. Dedryver, F.; Paillard, S.; Mallard, S.; Robert, O.; Trottet, M.; Nègre, S.; Verplancke, G.; Jahier, J. Characterization of genetic components involved in durable resistance to stripe rust in the bread wheat “Renan". Phytopathology 2009, 99, 968-973. [CrossRef]

23. Naruoka, Y.; Garland-Campbell, K.A.; Carter, A.H. Genome-wide association mapping for stripe rust (Puccinia striiformis f. sp. tritici) in US Pacific Northwest winter wheat (Triticum aestivum L.). Theor. Appl. Genet. 2015, 128, 1083-1110. [CrossRef] [PubMed]

24. Lu, Y.M.; Lan, C.X.; Liang, S.S.; Zhou, X.C.; Liu, D.; Zhou, G.; Lu, Q.L.; Jing, J.X.; Wang, M.N.; Xia, X.C.; et al. QTL mapping for adult-plant resistance to stripe rust in Italian common wheat cultivars Libellula and Strampelli. Theor. Appl. Genet. 2009, 119, 1349-1359. [CrossRef] [PubMed]

25. Hou, L.; Chen, X.M.; Wang, M.N.; See, D.R.; Chao, S.; Bulli, P.; Jing, J. Mapping a large number of QTL for durable resistance to stripe rust in winter wheat Druchamp using SSR and SNP markers. PLoS ONE 2015, 10, e0126794. [CrossRef] [PubMed]

26. Case, A.J.; Naruoka, Y.; Chen, X.M.; Garland-Campbell, K.A.; Zemetra, R.S.; Carter, A.H. Mapping stripe rust resistance in a Brundage $\times$ Coda winter wheat recombinant inbred line population. PLoS ONE 2014, 9, e91758. [CrossRef] [PubMed]

27. Suenaga, K.; Singh, R.P.; Huerta-Espino, J.; William, H.M. Microsatellite markers for genes Lr34/ Yr18 and other quantitative trait loci for leaf rust and stripe rust resistance in bread wheat. Phytopathology 2003, 93, 881-890. [CrossRef]

28. Ren, Y.; He, Z.H.; Li, J.; Lillemo, M.; Wu, L.; Bai, B.; Lu, Q.X.; Zhu, H.Z.; Zhou, G.; Du, J.Y.; et al. QTL mapping of adult-plant resistance to stripe rust in a population derived from common wheat cultivars Naxos and Shanghai 3/Catbird. Theor. Appl. Genet. 2012, 125, 1211-1221. [CrossRef]

29. Somers, D.J.; Isaac, P.; Edwards, K. A high-density microsatellite consensus map for bread wheat (Triticum aestivum L.). Theor. Appl. Genet. 2004, 109, 1105-1114. [CrossRef]

30. Maccaferri, M.; Zhang, J.; Bulli, P.; Abate, Z.; Chao, S.; Cantu, D.; Bossolini, E.; Chen, X.M.; Pumphrey, M.; Dubcovsky, J. A genome-wide association study of resistance to stripe rust (Puccinia striiformis f. sp. tritici) in a worldwide collection of hexaploid spring wheat (Triticum aestivum L.). G3: Genes Genomes. Genetics 2015, $5,449-465$.

31. Liu, J.; Chang, Z.; Zhang, X.; Yang, Z.; Li, X.; Jia, J.; Zhan, H.; Guo, H.; Wang, J. Putative Thinopyrum intermedium-derived stripe rust resistance gene Yr50 maps on wheat chromosome arm 4BL. Theor. Appl. Genet. 2013, 126, 265-274. [CrossRef] 
32. Lu, Y.; Wang, M.N.; Chen, X.M.; See, D.R.; Chao, S.M.; Jing, J. Mapping of Yr62 and a small-effect QTL for high-temperature adult-plant resistance to stripe rust in spring wheat PI 192252. Theor. Appl. Genet. 2014, 127, 1449-1459. [CrossRef]

33. Christopher, M.D.; Liu, S.; Hall, M.D.; Marshall, D.S.; Fountain, M.O.; Johnson, J.W.; Millus, E.A.; Garland-Campbell, K.A.; Chen, X.M.; Griffey, C.A. Identification and mapping of adult plant stripe rust resistance in soft red winter wheat VA00W-38. Crop Sci. 2013, 53, 871-879. [CrossRef]

34. Chen, X.M.; Line, R.F. Inheritance of stripe rust resistance in wheat cultivars used to differentiate races of Puccinia striiformis in North America. Phytopathology 1992, 82, 633-637. [CrossRef]

35. Zadoks, J.C.; Chang, T.T.; Konzak, C.F. A decimal code for the growth stages of cereals. Weed Res. 1974, 14, 415-421. [CrossRef]

36. Line, R.F.; Qayoum, A. Virulence, aggressiveness, evolution and distribution of races of Puccinia striiformis (the cause of stripe rust of wheat) in North America, 1968-1987. In Technical Volume 1788; United States Department of Agriculture: Washington, DC, USA, 1992; p. 44.

37. Chen, X.M.; Line, R.F. Gene action in wheat cultivars for durable, high-temperature, adult-plant resistance and interaction with race-specific, seedling resistance to Puccinia striiformis. Phytopathology 1995, 85, 567-572. [CrossRef]

38. Riede, C.R.; Anderson, J.A. Linkage of RFLP markers to an aluminum tolerance gene in wheat. Crop Sci. 1996, 36, 905-909. [CrossRef]

39. Wang, S.; Wong, D.; Forrest, K.; Allen, A.; Chao, S.; Huang, B.E.; Maccaferri, M.; Salvi, S.; Milner, S.G.; Cattivelli, L.; et al. Characterization of polyploid wheat genomic diversity using a high-density 90,000 single nucleotide polymorphism array. Plant Biotechnol. J. 2014, 12, 787-796. [CrossRef]

40. Van Ooijen, J.W. Multipoint maximum likelihood mapping in a full-sib family of an outbreeding species. Genet. Res. 2011, 93, 343-349. [CrossRef]

41. Kosambi, D.D. The estimation of map distance from recombination values. Ann. Eugen. 1943, 12, $172-175$. [CrossRef]

42. Voorrips, R.E. MapChart: Software for the graphical presentation of linkage maps and QTLs. J. Hered. 2002, 93, 77-78. [CrossRef]

43. Broman, K.W.; Wu, H.; Sen, S.; Churchill, G.A. R/qtl: QTL mapping in experimental crosses. Bioinformatics 2003, 19, 889-890. [CrossRef]

44. Arends, D.; Prins, P.; Jansen, R.C.; Broman, K.W. R/qtl: High-throughput multiple QTL mapping. Bioinformatics 2010, 26, 2990-2992. [CrossRef]

45. Qie, Y.M.; Liu, Y.; Wang, M.N.; Li, X.; See, D.R.; An, D.G.; Chen, X.M. Development, validation, and re-selection of wheat lines with pyramided genes $Y r 64$ and $Y r 15$ linked on the short arm of chromosome 1B for resistance to stripe rust. Plant Dis. 2018, 103, 51-58. [CrossRef] 